\title{
nombalina
}

(8)

Modelos financeiros de projectos mineiros: incorporação do risco e abordagens de avaliação
Autor(es):
Godinho, Pedro
Publicado por: Imprensa da Universidade de Coimbra; Laboratório de Radioactividade Natural da Universidade de Coimbra
persistente:
URI:http://hdl.handle.net/10316.2/36303
DOI:
DOl:http://dx.doi.org/10.14195/978-989-26-1009-2_15
Accessed : $\quad$ 26-Apr-2023 00:47:43

A navegação consulta e descarregamento dos títulos inseridos nas Bibliotecas Digitais UC Digitalis, UC Pombalina e UC Impactum, pressupõem a aceitação plena e sem reservas dos Termos e Condições de Uso destas Bibliotecas Digitais, disponíveis em https://digitalis.uc.pt/pt-pt/termos.

Conforme exposto nos referidos Termos e Condições de Uso, o descarregamento de títulos de acesso restrito requer uma licença válida de autorização devendo o utilizador aceder ao(s) documento(s) a partir de um endereço de IP da instituição detentora da supramencionada licença.

Ao utilizador é apenas permitido o descarregamento para uso pessoal, pelo que o emprego do(s) título(s) descarregado(s) para outro fim, designadamente comercial, carece de autorização do respetivo autor ou editor da obra.

Na medida em que todas as obras da UC Digitalis se encontram protegidas pelo Código do Direito de Autor e Direitos Conexos e demais legislação aplicável, toda a cópia, parcial ou total, deste documento, nos casos em que é legalmente admitida, deverá conter ou fazer-se acompanhar por este aviso.

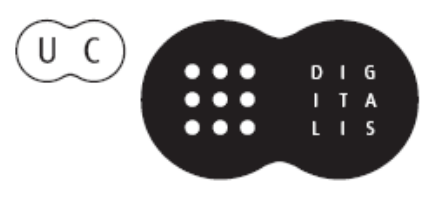




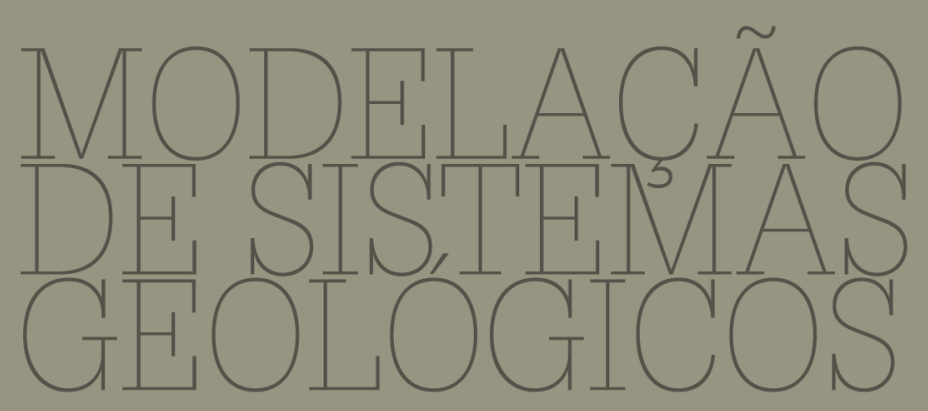

Homenagem ao Professor Doutor Manuel Maria Godinho

L.J.P.F. Neves, A.J.S.C. Pereira,

C.S.R. Gomes, L.C.G. Pereira,

A.O. TAVARES

IMPRENSA DA UNIVERSIDADE DE COIMBRA

CoImBra University Press 


\title{
MODELAÇÃO \\ DE SISTEMAS \\ GEOLÓGICOS
}

Homenagem ao Professor Manuel Maria Godinho

\section{Modelos financeiros de projectos mineiros: incorporação do risco e abordagens de avaliação}

\author{
Pedro Godinho ${ }^{1}$ \\ ${ }^{1}$ Faculdade de Economia da Universidade de Coimbra, PORTUGAL, pgodinho@fe.uc.pt
}

Palavras-chave: Avaliação de projectos mineiros, Opções reais

\section{Resumo}

Uma avaliação financeira realista de um projecto mineiro requer a correcta incorporação do risco, e a consideração da estratégia de gestão a ser seguida. Os modelos de opções reais permitem incorporar, de forma teoricamente sustentada, o risco e a flexibilidade operacional na avaliação financeira dos projectos, permitindo ainda obter a estratégia óptima de gestão do projecto. No presente artigo apresenta-se uma perspectiva geral sobre a incorporação do risco em modelos financeiros de projectos mineiros. Uma vez que o risco e a flexibilidade operacional conduzem geralmente a uma maior complexidade analítica, é também importante perceber como é possível formalizar estes modelos e obter as correspondentes soluções. Assim, apresentam-se também as principais abordagens de avaliação de modelos financeiros de projectos mineiros.

Key-words: Valuation of mining projects, Real options

\section{Abstract}

The proper valuation of a mining project must take into account both the project risk and the management strategy that will be followed. Real options analysis allows a theoretically sound incorporation of risk and operational flexibility in the financial evaluation of investment projects, also providing the optimal strategy for the management of the project. This paper presents a general view on the incorporation of risk in financial models of mining projects. Since accounting for risk and operational flexibility leads to greater model complexity, it is important to understand how such models may be built, and how the solutions may be calculated. Therefore, the most important valuation approaches are also presented.

\section{Introdução}

O risco é uma componente importante dos projectos mineiros. Na presença de risco, os gestores podem tomar decisões que respondam à ocorrência de 
acontecimentos incertos. Estas decisões podem permitir minorar as consequências de desenvolvimentos negativos (e.g., a suspensão temporária das operações caso ocorra uma descida significativa nos preços dos recursos extraídos) ou majorar os benefícios de desenvolvimentos positivos (e.g., a exploração de novas reservas com no caso de subida dos preços dos recursos geológicos).

Na presença de risco e da possibilidade de tomar decisões contigentes a acontecimentos incertos, as abordagens clássicas, que pressupõem uma gestão passiva, não permitem uma avaliação financeira realista dos projectos de investimento. Para estas situações foram desenvolvidos modelos de Análise de Opções Reais (AOR) que permitem a incorporação do risco e da flexibilidade operacional na avaliação dos projectos. Estes modelos determinam simultaneamente o valor do projecto e a estratégia óptima para a sua gestão, sendo assim a obtenção de soluções mais complexa do que nos modelos clássicos. Neste artigo procura-se dar uma perspectiva geral sobre as formas utilizadas para incorporar o risco em modelos de opções reais aplicados a projectos mineiros, e sobre as abordagens de avaliação destes modelos

$\mathrm{O}$ artigo encontra-se estruturado da seguinte forma. Depois desta introdução, o capítulo 2 discute a incorporação de diferentes riscos em modelos de AOR de projectos mineiros. No Capítulo 3 irão apresentar-se as principais abordagens de avaliação utilizadas para obter soluções óptimas para estes modelos. Finalmente, o Capítulo 4 apresenta os comentários finais.

\section{Modelação do Risco nos Projectos Mineiros}

$\mathrm{O}$ risco, e a forma de reagir perante diferentes eventos aleatórios, podem influenciar significativamente o valor dos projectos de investimento. Os modelos financeiros tendem a enfatizar o risco do preço dos outputs, ou seja, o risco relativo ao preço de venda dos produtos do projecto. No caso de projectos mineiros, o risco relativo aos custos de operação e os riscos geológico e técnico, relativos à dimensão e características da mina a explorar, revestem-se também de grande importância. Finalmente, também o risco político pode ser relevante nalguns investimentos mineiros.

\section{O Risco do Preço dos Recursos Geológicos}

Considera-se geralmente que o trabalho de Tourinho (1979) constitui a primeira aplicação de AOR a projectos de extracção de recursos naturais. 
Tourinho considera a exploração de petróleo, e modela a evolução do preço deste $(S)$ como um Movimento Browniano Geométrico (MBG):

$$
d S=\mu \cdot S \cdot d t+\sigma \cdot S \cdot d z
$$

em que $d t$ é uma variação infinitesimal do tempo, $d z$ é o incremento de um processo de Wiener padrão, seguindo uma distribuição normal com média nula e variância $d t^{l}, d S$ é a variação instantânea do preço do petróleo, $\mu$ é o parâmetro drift que representa a tendência média de evolução do preço, e $\sigma$ é o parâmetro da volatilidade deste preço. Tourinho assume que a variação média do preço, $\mu$, corresponde exactamente à taxa de rentabilidade de investimentos com risco idêntico transaccionados em mercados de capitais competitivos. Este pressuposto constitui uma importante fragilidade do modelo de Tourinho (ver, por exemplo, Adkins e Paxson, 2008). Com efeito, é hoje aceite que o processo de variação do preço deste tipo de recursos incorpora uma convenience yield que reflecte os benefícios e custos da sua detenção física. Brennan e Schwartz (1985) descrevem esta convenience yield como o fluxo de serviços que advêm ao proprietário do recurso físico, mas não ao detentor de um contrato que permita o recebimento futuro do recurso (por exemplo, a possibilidade de obter lucro em situações de falta temporária do recurso, ou de beneficiar de diferenças de preço entre diferentes locais).

A convenience yield é modelada de forma diversa por diferentes autores. Brennan e Schwartz (1985), num artigo seminal de aplicação de AOR à avaliação de uma exploração mineira, modelam o processo de evolução do preço do recurso geológico de forma semelhante a,

(2.1), mas assumem que a convenience yield é uma função do preço e admitem que $\mu$ possa ser estocástico. Por forma a obterem um modelo analiticamente tratável, estes autores assumem nalguns casos que a convenience yield é uma proporção constante do preço do recurso. Este modelo de variação de preços, em que o preço do recurso segue um processo semelhante a, (2.1) com convenience yield proporcional ao preço, é seguido por vários outros autores na modelação de preços de metais, especialmente no caso de metais preciosos. Moyen et al. (1996), Cortazar e Casassus (1999) e Cortazar et al. (2001) usam um tal processo para modelar o preço do cobre; Kelly (1998), Colwell et al. (2003), Abdel Sabour e Poulin (2006), Costa Lima e Suslick (2006) e Dimitrakopoulos e Abdel Sabour (2007) usam-no para o ouro; Shafiee et al.

\footnotetext{
${ }^{1}$ Para mais detalhes sobre os processos de Wiener ver, por exemplo, Dixit e Pyndick (1994).
} 
(2009) usam-no para o zinco; Abdel Sabour e Poulin usam-no igualmente para a prata e paládio.

Schwartz (1997) propõe um modelo de preços em que existe reversão à média, isto é, em que os preços oscilam aleatoriamente mas tendem a retornar a um valor médio de longo prazo. Schwartz nota que por vezes o preço à vista de um recurso é tão incerto que se torna preferível usar, como aproximação, o preço do contrato futuro com maturidade mais próxima. Schwartz estima modelos para os preços do petróleo, cobre e ouro, usando filtros de Kalman - uma metodologia que permite estimar os modelos assumindo que os preços à vista dos recursos não são directamente observados, e considerando apenas os preços de contratos para entrega futura e a sua relação com os preços à vista. Schwartz encontra reversão à média estatisticamente significativa nos preços do petróleo e cobre, não a encontrando no preço do ouro. Num outro estudo, também Slade (2001) encontra reversão à média no processo de variação dos preços do cobre. Estes resultados têm levado vários autores a usar processos MBG para modelar a evolução dos preços de metais preciosos, e processos com reversão à média para modelar os preços de metais não preciosos.

$\mathrm{O}$ processo de preços com reversão à média, considerado por Schwartz (1997), assume que o preço médio de longo prazo, a convenience yield, a volatilidade, a taxa de juro sem risco e a velocidade de reversão são determinísticas. Este processo é denominado processo de Ornstein-Uhlenbeck geométrico, podendo definir-se como:

$$
d S=\eta \cdot(\ln (\bar{S})-\ln (S)) \cdot S \cdot d t+\sigma \cdot S \cdot d z,
$$

em que $S$ é o preço cuja evolução se está a modelar, $\eta$ é velocidade de reversão à média, $\bar{S}$ é o preço médio de longo prazo, e $\sigma$ é a volatilidade do preço. Este processo é adoptado por vários autores: Cortazar e Casassus (1998), Sagi (1998), Slade (2001), Abdel Sabour e Poulin (2006) e Caldentey et al. (2007) usam este tipo de processo para o preço do cobre; Abdel Sabour e Poulin usam-no igualmente para os preços do níquel, cobalto e platina.

Gibson e Schwartz (1989) estudam o comportamento do preço do petróleo, concluindo que a convenience yield deste recurso segue um processo de reversão à média. Baseados neste resultado, Gibson e Schwartz (1990) propõem um modelo de evolução do preço baseado numa convenience yield estocástica com reversão à média. Brennan (1991) também usa um modelo com convenience yield estocástica, semelhante ao de Gibson e Schwartz, estimando-o para os preços de vários recursos, incluindo quatro metais: ouro, prata, platina e cobre. $\mathrm{O}$ autor compara os 
resultados do modelo com os resultados de modelos que assumem que a convenience yield é função do preço à vista dos metais (incluindo o modelo usado por Brennan e Schwartz, 1985, em que esta representa uma proporção constante do preço do metal). Brennan conclui que, se se excluir em período em que existiu uma forte influência de uma intervenção especulativa atípica no mercado da prata $^{2}$, o modelo em que a convenience yield é uma proporção constante do preço do metal obtém bons resultados na descrição do processo de evolução dos preços dos metais preciosos; já o modelo de reversão à média da convenience yield se mostra bastante mais ajustado à descrição do processo de evolução dos preços do cobre. Um modelo deste tipo, em que a convenience yield apresenta reversão à média, é usado por Moel e Tufano (1999) para modelar a dinâmica conjunta dos preços do cobre e zinco, incorporando-se ainda uma correlação constante entre os preços destes metais. Também Tsekrekos et al. (2003) recorrem a um modelo deste tipo para o preço do cobre.

Cortazar e Schwartz (2003) propõem um modelo em que o retorno de longo prazo do preço dos recursos e a convenience yield seguem um processo de reversão à média, e aplicam-no ao preço do petróleo. Cortazar et al. (2008) utilizam este modelo para os preços do cobre, no contexto da avaliação de uma mina. Outros modelos da evolução dos preços de recursos têm vindo a ser propostos, mas a sua aplicação em modelos de avaliação ainda não tem uma expressão muito forte. Estes incluem Casassus e Collin-Dufresne (2005) e Trolle e Schwartz (2009), entre outros.

No caso de o recurso geológico estar cotado numa divisa diferente da usada no país de origem da empresa que empreende o projecto, o risco de taxa de câmbio torna-se uma componente relevante do risco do preço do recurso. Numa tal situação, para além do processo de variação do preço do recurso, será ainda necessário definir um processo para a variação da taxa de câmbio. É isso que é feito por Dimitrakopoulos e Sabour (2007), que consideram a extracção de ouro numa mina Australiana, estando o metal cotado em dólares dos Americanos. Os autores usam um MBG para modelar a variação do preço do ouro, em dólares Americanos, e um processo de reversão à média para a taxa de câmbio entre o dólar Australiano e o dólar Americano.

\footnotetext{
2 Mais concretamente, a intervenção dos irmãos Hunt. Apesar da posição dos irmãos Hunt ter tido o seu colapso no final de Março de 1980, Brennan considerou que a perturbação do mercado que estes causaram se estendeu para além desta data, considerando atípico (e removendo da amostra) o período que vai de Setembro de 1979 até Maio de 1981.
} 


\section{O Risco Associado aos Custos de Operação}

Os custos de operação têm grande importância para a rentabilidade dos projectos mineiros, podendo o risco relativo a estes custos estar ou não relacionado com incerteza sobre algumas características da mina a explorar. Neste trabalho, o risco nos custos que está associado a incerteza sobre características da mina será considerado risco técnico, e analisado em conjunto com o risco geológico na Secção 2.3. A presente secção tratará do risco associado aos custos de operação que não tem relação directa com as características da mina.

A generalidade dos autores assume que o risco associado aos custos de operação de uma mina é muito inferior ao risco do preço dos recursos extraídos, ignorando o primeiro tipo de risco. Desta forma, grande parte dos trabalhos publicados assume que os custos de exploração são conhecidos com certeza. Existem, no entanto, alguns autores que modelam explicitamente o risco associado aos custos. Esta modelação é efectuada definindo um processo de variação do custo, de forma semelhante ao que se passa com o risco do preço dos recursos extraídos. Um exemplo da utilização desta abordagem é Slade (2001). A autora utiliza dados reais para estimar o custo de operação de minas de cobre canadianas, concluindo que o custo apresenta reversão à média. A autora parte de um modelo que considera que o custo pode estar correlacionado com o preço do metal (cobre), mas não encontra indícios de correlação nos dados, usando por isso um coeficiente de correlação nulo. Costa Lima e Suslick (2006) modelam os custos de operação de uma mina de ouro como um processo MBG correlacionado com o processo do preço do ouro. Moyen et al. (1996) não consideram directamente os custos de operação, mas sugerem que estes podem ser incluídos substituindo o preço do metal pela margem unitária, isto é, a diferença entre o preço unitário e o custo unitário. Note-se que para tal é necessário que esta diferença siga um processo idêntico ao usado para o preço do metal, que no caso destes autores é um MBG.

\section{O Risco Geológico e Técnico}

O risco geológico pode influenciar o valor dos investimentos em minas quer na fase de exploração quer na fase de prospecção. A generalidade dos modelos considera que a prospecção já foi concluída, e por isso o risco relevante é apenas o relativo à fase de exploração. Muitas vezes, as características da mina a explorar não são conhecidas a priori, e destas dependerá não só a quantidade de metal que pode ser extraído, mas também os custos de operação - falar-se-á, neste último caso, em risco técnico, e este estará geralmente associado aos mesmos factores que definem o risco 
geológico. Estes dois tipos de risco, técnico e geológico, influenciam decisivamente a rentabilidade da exploração mineira mas, tal como ocorre com o risco relativo aos custos de operação, são frequentemente ignorados. Assume-se, geralmente, que a quantidade de metal a extrair é conhecida com certeza a priori, e que os custos não dependem de características da mina sujeitas a incerteza. Existem, no entanto, alguns autores que incorporam o risco geológico e técnico nos modelos.

Martzoukos (2000) modela o risco na fase de prospecção através de um processo de salto. $\mathrm{O}$ autor considera que a empresa tem a oportunidade de empreender actividades de prospecção, cada uma delas com um custo definido, e que destas resultam saltos no valor esperado do projecto, decorrentes de nova informação sobre a dimensão e/ou qualidade das reservas.

Ao analisar a fase de exploração da mina, alguns autores modelam directamente as características geológicas e técnicas recorrendo a distribuições de probabilidades. Este é o caso de Moel e Tufano (1999), que definem três cenários para as características da mina, especificando para cada um deles a quantidade total de mineral, a quantidade de cada metal obtida anualmente e os custos anuais de operação. A cada um dos cenários é atribuída uma probabilidade, considerando-se assim que esta distribuição discreta de probabilidades sobre os cenários representa o risco geológico do investimento. Dimitrakopoulos et al. (2002) e Dimitrakopoulos e Abdel Sabour (2007) utilizam uma técnica, designada por "simulação condicional", que permite quantificar a incerteza e variabilidade espacial de variáveis geológicas, tais como a quantidade de mineral e teor de metal, com base na informação geológica disponível.

Outros autores definem um processo estocástico para as características geológicas da mina, geralmente considerando apenas as reservas. É este o caso de Slade (2001). A autora modela as reservas remanescentes em minas de cobre através de um processo estocástico com uma componente determinística que tem em conta o minério extraído e os valores desfasados dos custos de operação e preços do cobre. Slade justifica a utilização deste processo pela definição de reservas como depósitos de minerais que são economicamente viáveis aos preços e custos actuais, pelo que estes preços e custos podem alterar a dimensão das reservas. A componente estocástica é justificada pela aquisição de nova informação sobre o depósito de mineral à medida que o tempo passa. Ao estimar os parâmetros do processo a partir de dados de minas e cobre Canadianas, a autora conclui que os custos de operação e preços desfasados não apresentam significância estatística, 
optando assim por um processo com variância constante e independente dos preços do cobre e dos custos de operação.

Num modelo que considera a prospecção e exploração de uma mina, Cortazar et al. (2001) consideram que a prospecção se divide em várias fases, caracterizadas por probabilidades de sucesso e insucesso. Cada uma das fases apresenta um custo e, caso alguma tenha insucesso, o projecto é terminado (o insucesso representa a inexistência de reservas economicamente viáveis). Os autores consideram que, no caso de sucesso em todas as fases de prospecção, a mina pode ter diferentes características. Estas são definidas por um conjunto representativo de tipos de mina, cada um destes com uma determinada probabilidade de ocorrência. Cada tipo de mina é caracterizado por um conjunto de parâmetros: reservas totais, tempo e custo necessários para o desenvolvimento (isto é, para preparar a mina para o início da extracção), ritmo de extracção e custos associados à extracção e ao encerramento temporário da mina.

\section{O Risco Político}

A exploração mineira pode estar sujeita a riscos decorrentes de alterações das condições políticas, como por exemplo o risco de expropriação. Este risco poderá ser significativo nalguns países, e pouco relevante noutros. A generalidade dos autores que considera projectos mineiros não tem em conta este risco, mas a sua inclusão pode ser importante, especialmente quando a exploração é feita por empresas multinacionais e as minas são localizadas em países politicamente instáveis.

Mahajan (1990) e Schwartz e Trolle (2010) modelam a expropriação como uma decisão racional por parte do governo do país onde o projecto é empreendido. O governo detém uma opção de expropriação que pode exercer a qualquer momento, e fá-lo-á se tal vier a ser óptimo. Quando o governo exerce essa opção, a empresa perde o projecto, podendo vir a receber uma compensação. Para determinar quando é que é óptimo para o governo proceder à expropriação, é necessário conhecer os custos que esta apresenta, nomeadamente "custos reputacionais" e custos esperados de eventuais medidas de retaliação por outros países. Outros autores modelam os impactes do risco político como acontecimentos aleatórios exógenos ao projecto. Feils e Şabac (2000) propõem um modelo que tem em conta o risco de expropriação e outros riscos associados ao país em que é feito o investimento. A utilização deste modelo requer a definição de uma probabilidade de expropriação e de uma taxa esperada de variação dos fluxos de caixa devido a outros riscos políticos. A dificuldade em estimar os 
parâmetros necessários torna problemática a aplicação destes modelos a projectos concretos.

Cortazar et al. (2001) consideram o risco de expropriação de uma mina, assumindo que esta corresponde à perda total do investimento, sem compensação, para a empresa que o empreende, e que a sua probabilidade se mantém constante. A incorporação deste risco no valor do projecto requer a definição da probabilidade de expropriação por unidade de tempo, baseando-se na utilização de processos de Poisson para modelar acontecimentos passíveis de provocar saltos no valor de um projecto (ver, por exemplo, Dixit e Pindyck, 1994, ponto 5B do capítulo 3). Usando uma filosofia semelhante, Clark (1997) modela acontecimentos políticos que podem causar uma redução do valor do projecto através de um processo de Poisson. Clark e Tunaru (2003; 2008) estendem esta abordagem a múltiplas fontes de risco político. Estas abordagens apresentam grande flexibilidade, mas a dificuldade de especificar de forma razoável os parâmetros necessários torna a sua aplicação difícil.

\section{Abordagens de Avaliação}

Nos modelos de AOR, os autores procuram muitas vezes obter soluções analíticas de forma fechada, que possam ser aplicadas a todas as situações que se enquadrem nos pressupostos considerados. No entanto, os modelos mais realistas exigem, frequentemente, pressupostos complexos que não permitem obter uma solução com estas características, obrigando à utilização de métodos aproximativos. Neste capítulo apresentam-se as principais abordagens utilizadas para definir modelos de AOR de projectos mineiros, e para obter as respectivas soluções.

\section{Equações Diferenciais com Solução Analítica de Forma Fechada}

Os modelos de opções reais são muitas vezes definidos por forma a obter equações diferenciais com condições de fronteira que possam conduzir a soluções analíticas de forma fechada. Num artigo seminal de aplicação de AOR à avaliação de uma mina de cobre, Brennan e Schwartz (1985) obtêm solução analítica de forma fechada apenas para o caso particular em que a quantidade de cobre existente na mina é infinita. Dixit e Pyndick (1994, capítulo 7) apresentam um modelo com muitas semelhanças com o de Brennan e Schwartz e, para obterem solução analítica de forma fechada, assumem também que a mina pode ser explorada a um ritmo constante para sempre (ignorando que as reservas existentes são limitadas). Quando se consideram problemas em que as reservas do recurso a extrair são limitadas 
não é, geralmente, possível obter soluções com forma fechada, a não ser com significativas simplificações adicionais. Costa Lima e Suslick (2006) obtêm uma solução de forma fechada para o valor de uma mina de ouro, mas consideram apenas a flexibilidade operacional relativa ao momento de iniciar o investimento, bem como um processo simplificado de variação do valor do projecto.

\section{Equações Diferenciais com Solução Numérica}

Nos modelos de opções reais de projectos mineiros, é geralmente impossível obter soluções de forma fechada quando se considera que a quantidade de metal que pode ser extraída é finita. Neste caso, o modelo é por vezes formalizado através de equações diferenciais, mas a solução destas é obtida por métodos numéricos. O método numérico mais usado para resolver as equações diferenciais é o das diferenças finitas. Neste método, as derivadas são aproximadas, em cada ponto, por diferenças finitas de valores. (ver, por exemplo, Trigeorgis, 1996, capítulo 10).

A determinação das equações diferenciais que definem o valor do projecto, e sua resolução pelo método das diferenças finitas, proporcionam uma importante flexibilidade na modelação. Davis (1998) usa este método para avaliar o projecto de exploração de uma mina de um metal precioso com alguns parâmetros que variam ao longo da vida deste (e.g. a volatilidade do projecto). Cortazar e Casassus (1999), Cortazar et al. (2001) e Colwell et al. (2003) utilizam também o método das diferenças finitas para obter soluções numéricas para modelos de avaliação de projectos mineiros formulados com recurso a equações diferenciais.

\section{Lattices}

As árvores de decisão, e estruturas que nelas se baseiam, têm vindo a ser frequentemente aplicadas a projectos de investimento. Em AOR são muito usadas estruturas designadas por lattices, que se podem considerar árvores em que existe recombinação de caminhos: isto é, em que podem existir vários caminhos diferentes entre a origem e um dado nodo. A Figura 1 apresenta um exemplo do tipo de lattice mais simples, a lattice binomial, em que cada nodo tem dois descendentes. Outras estruturas, em que cada nodo tem mais do que dois descendentes, têm também vindo a ser propostas (e.g. Boyle et al., 1989). 


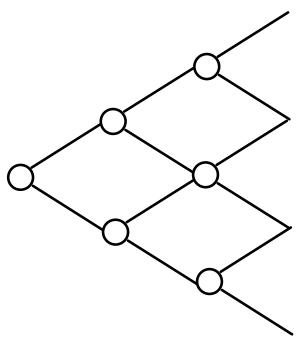

Figura 1. Estrutura de uma lattice binomial com três intervalos de tempo

As lattices modelam directamente o processo de variação das variáveis de estado de que depende o valor do projecto. Desta forma, as lattices permitem efectuar, de forma simples a ligação entre os estados do projecto e os correspondentes valores e decisões óptimas. Tal torna a sua utilização intuitiva, o que constitui um importante benefício da sua utilização.

No caso de projectos de exploração mineira, a variável de estado relevante é usualmente o preço do recurso a extrair ${ }^{3}$. Os níveis da lattice correspondem a sucessivos momentos no tempo e, dentro em cada nível, os diferentes nodos correspondem aos possíveis preços que se estão a considerar para esse momento. Atendendo a que tipicamente os preços variam em tempo contínuo e podem assumir uma gama de valores mais vasta do que a considerada pelas lattices, estas correspondem a uma aproximação do processo de preços. Esta aproximação deve ser feita de forma a que o processo considerado na lattice se aproxime o mais possível do verdadeiro processo de variação dos preços. Algumas formas de efectuar esta aproximação podem encontrar-se em Cox et al. (1979) ou Trigeorgis (1991).

Quando existe recombinação de caminhos, o crescimento do número de nodos das lattices é tipicamente polinomial com o número de níveis destas (linear no caso de lattices que apenas considerem uma variável de estado). No entanto, diversos processos de variação das variáveis de estado não permitem a recombinação de caminhos - este é o caso de processos em que a volatilidade não é constante (nem é possível uma transformação que a torne constante). Nesta situação, as lattices transformam-se em árvores, e o número de nodos cresce exponencialmente com o número de níveis

\footnotetext{
${ }^{3}$ Nalgumas circunstâncias pode ser preferível a utilização, como variável de estado, do valor do projecto na ausência de flexibilidade operacional (e.g. Davis, 1998).
} 
considerado. Em tais casos, pode não ser praticável a utilização de um número de níveis suficientemente elevado para permitir uma boa aproximação ao processo de variação do valor das variáveis de estado, sendo preferível utilizar outras abordagens.

Uma lattice corresponde sempre a uma árvore de decisão. As diferenças principais entre as duas estruturas correspondem a algumas simplificações da representação que tornam a lattice menos complexa: a não representação dos nodos de decisão e a recombinação dos nodos. Assim, em cada nodo da lattice está subjacente a escolha de uma acção válida para o projecto em causa: por exemplo, se o projecto ainda não tiver sido iniciado, as acções válidas poderão ser "Iniciar" e "Não iniciar"; se estiver em operação, poderão ser "Manter em operação", "Suspender" e "Abandonar". Estas acções contribuem também para definir o estado do projecto, tendo implicações nas escolhas óptimas a fazer no futuro, e mesmo nas acções disponíveis no futuro (por exemplo, não se pode manter em operação um projecto que já foi abandonado).

A avaliação das lattices segue os princípios da programação dinâmica, processando-se de forma semelhante à avaliação de árvores de decisão. Começa por se definir, para o último nível, qual a decisão óptima para cada possível estado do projecto; os valores óptimos do último nível são usados para determinar as decisões óptimas do penúltimo nível, e assim sucessivamente até se determinar a decisão óptima no primeiro nível, e o correspondente valor.

Diversos autores aplicam lattices à avaliação de projectos mineiros. Moyen et al. (1996), Hall e Nichols (2007), Kelly (1998) e Shaffiee et al. (2009) recorrem a lattices binomiais para avaliar minas de cobre (nos dois primeiros casos), ouro (no terceiro) e zinco (no último), usando como variáveis de estado o preço dos respectivos metais. Kamrad e Ernst (2001) usam uma lattice binomial como base de avaliação de um modelo em que o ritmo de extracção de metal de uma mina pode ser ajustado ao longo do tempo; a variável de estado utilizada é o preço do metal. Davis (1998) utiliza uma lattice binomial para avaliar uma reserva de um metal precioso, usando como variável de estado o valor do projecto na ausência de flexibilidade operacional. Slade (2001) utiliza lattices com 3 variáveis de estado - preço, custo e reservas remanescentes - para avaliar minas de cobre. A autora define, para cada variável de estado, um processo de evolução binomial. Uma vez que a volatilidade estimada para o preço do cobre e custo de exploração não são constantes, a autora recorre a uma transformação das variáveis que conduz a um parâmetro da volatilidade constante. 
Outras estruturas conceptualmente semelhantes têm vindo a ser usadas por outros autores. Martzoukos (2000) utiliza estruturas semelhantes a lattices mas com formato rectangular, em vez de triangular. Nestas estruturas, o autor modela o valor potencial das receitas anuais do projecto durante o período de prospecção de uma mina. Estas estruturas correspondem a uma "expansão" de uma lattice, necessária devido ao facto de $\mathrm{o}$ autor assumir que a informação recolhida nas actividades de prospecção pode provocar saltos no valor das receitas esperadas do projecto.

\section{Simulação de Monte Carlo}

A simulação de Monte Carlo é uma técnica muito usada em análise de investimentos. Numa simulação de Monte Carlo é gerado, de forma aleatória, um grande número de caminhos relativos à evolução das variáveis de estado estocásticas relevantes. Em cada momento, os valores de cada variável dependem de amostras de distribuições aleatórias, dos valores anteriores da variável e/ou dos valores contemporâneos ou anteriores das restantes variáveis.

Quando apenas é necessário considerar decisões no final dos projectos, a utilização desta técnica é simples: as variáveis são simuladas até ao final do projecto, são aí definidas as decisões óptimas em cada caminho, e o valor do projecto com essas decisões óptimas é actualizado para o momento de análise. É este o procedimento seguido por Moel e Tufano (1999), para avaliação de uma mina de cobre e zinco. Os autores efectuam a simulação de um período de exploração em que se irá estimar a dimensão das reservas, e consideram apenas a decisão de iniciar ou não o projecto no final deste período. Simulando a evolução dos preços dos metais até ao final do período, os autores obtêm as decisões óptimas para cada cenário da dimensão das reservas, conseguindo desta forma avaliar o projecto.

Esta lógica não se adequa de forma simples a activos, financeiros ou reais, cujo valor dependa das decisões a tomar ao longo da vida destes, como é o caso de projectos em que possam ser tomadas decisões ao longo do tempo. Com efeito, o valor destes activos depende das decisões óptimas, mas as consequências das decisões tomadas em cada período dependem de decisões futuras. Assim, não é possível determinar quais são as decisões óptimas no momento em que os valores das variáveis estão a ser gerados no processo de simulação.

Apesar desta dificuldade em aplicar simulação de Monte Carlo à avaliação de projectos de investimento, há características desta técnica que a tornam particularmente atractiva. Uma destas características é a flexibilidade, permitindo a utilização de processos estocásticos complexos 
para a evolução das variáveis de estado. Outra é o facto de permitir evitar a "maldição da dimensionalidade" (curse of dimensionality): a complexidade da aplicação de métodos numéricos baseados em lattices ou de resolução de equações diferenciais cresce muito rapidamente com o número de variáveis de estado (geralmente é exponencial neste número de variáveis de estado); já a complexidade da simulação de Monte Carlo aumenta, tipicamente, de forma linear com o número de variáveis de estado, permitindo a resolução de problemas em que o número destas variáveis é elevado. Assim, têm vindo a ser propostas várias adaptações que permitem usar simulação de Monte Carlo para avaliar activos cujo valor depende de decisões que podem ser tomadas em vários períodos.

Longstaff e Schwartz (2001) propuseram um método, designado por Least-Squares Monte Carlo (LSMC), aplicável quando podem ser tomadas decisões durante todo o tempo de vida dos activos em avaliação. Neste método, começam por se simular os valores das variáveis de estado estocásticas para todo o tempo de vida dos activos. No último período de vida do activo não existem mais decisões posteriores, pelo que é possível determinar as decisões óptimas, e o valor do activo, em cada caminho e para cada possível estado em que este se encontre. No penúltimo período de vida do activo, é ajustado um modelo econométrico que permite, para cada possível estado e decisão, escrever o valor do activo como função das variáveis de estado. Este modelo é construído com base nos valores do último período (neste momento já determinados), e a sua aplicação permite determinar o valor esperado do activo em cada caminho, para cada estado e para cada possível decisão. Desta forma é possível determinar as decisões óptimas em cada caminho, bem como o correspondente valor do activo. Este procedimento vai sendo sucessivamente aplicado aos períodos anteriores, até se atingir o período inicial de avaliação. Assim determina-se não só o valor do activo, mas as regras óptimas relativas às decisões a tomar.

Este método tem vindo a ser usado na avaliação de projectos mineiros. Tsekrekos et al. (2003), Abdel Sabour e Poulin (2006) e Cortazar et al. (2008) usam LSMC para avaliar uma mina de cobre, assumindo diferentes processos para a variação do preço do metal. Abdel Sabour e Poulin aplicam ainda LSMC a um segundo caso de avaliação de uma mina de onde são extraídos múltiplos metais: ouro, prata, paládio, níquel, cobre, cobalto e platina. Dimitrakopoulos e Abdel Sabour (2007) utilizam LSMC para avaliar uma mina de ouro na Austrália, considerando incerteza no preço do ouro e nas taxas de câmbio, bem como incerteza geológica. 


\section{Nota Final}

As últimas três décadas viram aparecer uma extensa literatura sobre a incorporação do risco e flexibilidade operacional na avaliação de projectos de investimento: os modelos de opções reais. Devido às características dos projectos mineiros, o risco e a flexibilidade de gestão desempenham nestes um papel importante, e muitos modelos foram desenvolvidos para estes projectos. Neste artigo procurou dar-se conta das principais formas de incorporação de risco que têm vindo a ser consideradas em modelos financeiros de projectos mineiros, bem como das abordagens seguidas para formalizar e obter soluções para estes modelos.

\section{Bibliografia}

Abdel Sabour, S.A., Poulin R. (2006) -Valuing Real Capital Investments using the Least Squares Monte Carlo Method. The Engineering Economist; 51 (2), 141-160.

Adkins, R., Paxson, D. (2008) - The Tourinho Model: Neglected Nugget or Receding Relic. Proceedings of the 12th Annual International Conference on Real Options: Theory meets Practice, Rio de Janeiro, Brasil, 9-12 de Julho de 2008.

Boyle, P.P., Evnine, J., Gibbs, S. (1989) - Numerical Evaluation of Multivariate Contingent Claims. The Review of Financial Studies; 2 (2), 241-250.

Brennan, M.J. (1991) - The Price of Convenience and the Valuation of Commodity Contingent Claims in Lund, D. e Oksendal, B. (eds.), Stochastic Models and Option Values Applications to Resources, Environment and Investment Problems, Elsevier, Amsterdam, 33-71.

Brennan, M.J., Schwartz, E.S. (1985) - Evaluating Natural Resource Investments. Journal of Business; 58 (2), 135-157.

Casassus, J., Collin-Dufresne, P. (2005) - Stochastic Convenience Yield Implied from Commodity Futures and Interest Rates. Journal of Finance; 60 (5), 2283-2331.

Caldentey R., Epstein, R., Sauré, D. (2007) Optimal Exploitation of a Nonrenewable Resource. Working paper, Stern School, New York University.

Clark. E. (1997) - Valuing Political Risk. Journal of International Money and Finance; 16 (3), 477-490.

Clark. E., Tunaru, R. (2003) - Quantification of Political Risk with Multiple Dependent Sources. Journal of Economics and Finance; 27 (1), 125-135.

Clark. E., Tunaru, R. (2008) - Modelling Stochastic Political Risk for Capital Budgeting: Currency Crises. Banque et Marchés; 95, 45-56.

Colwell, D., Henker, T., Ho, J., Fong, K.. (2003) - Real Option Valuation of Australian Gold Mines and Mining Companies. The Journal of Alternative Investments; 6 (1), 23-38.

Cortazar, G., Casassus, J. (1998) - Optimal Timing of a Mine Expansion: Implementing a Real Options Model. The Quarterly Review of Economics and Finance; 38 (3), 755-769.

Cortazar, G., Casassus, J. (1999) - A Compound Option Model for Evaluating Multi Stage Natural Resource Investments in M. Brennan e L. Trigeorgis (eds.), Project Flexibility, Agency, and Competition, Oxford University Press, New York, 205-223.

Cortazar, G., Schwartz, E.S. (2003) - Implementing a Stochastic Model for Oil Futures Prices. Energy Economics; 25 (3), 215-238.

Cortazar, G., Schwartz, E.S., Casassus, J. (2001) - Optimal Exploration Investments under Price and Geological-Technical Uncertainty: A Real Options Model. R\&D Management; 31 (2), 181-189. 
Cortazar, G., Gravet, M., Urzua, J. (2008) - The Valuation of Multidimensional American Real Options using the LSM Simulation Method. Computers and Operations Research; 35 (1), 113-129.

Costa Lima, G., Suslick, S. (2006) - Estimating the Volatility of Mining Projects Considering Price and Operating Cost Uncertainties. Resources Policy; 31 (2), 86-94.

Cox, J.C., Ross, S.A., Rubinstein, M. (1979) - Option Pricing: A Simplified Approach. Journal of Financial Economics; 7 (3), 229-263.

Davis, G.A. (1998) - Estimating Volatility and Dividend Yield when Valuing Real Options to Invest or Abandon. The Quarterly Review of Economics and Finance; 38 (3), 725-754.

Dimitrakopoulos, R.G., Farrel, C.T., Godoy, M. (2002) - Moving Forward from Traditional Optimization. Transactions of the Institute of Mining and Metallurgy (Section A: Min. Tecnol.); 111, A82-A88.

Dimitrakopoulos, R.G., Abdel Sabour, S.A. (2007) - Evaluating Mine Plans under Uncertainty: Can the Real Options Make a Difference?. Resources Policy; 32 (3), 116-125.

Dixit, A.K., Pindyck, R.S. (1994) - Investment under Uncertainty. Princeton University Press, New Jersey.

Feils, D., Şabac, F. (2000) - The Impact of Political Risk on the Foreign Direct Investment Decision: A Capital Budgeting Analysis". The Engineering Economist; 45 (2), 129-143.

Gibson, R., Schwartz, E.S. (1989) - Valuation of Long Term Oil Linked Assets. Anderson Graduate School of Management, UCLA, Working Paper, \#6-89.

Gibson, R., Schwartz, E.S. (1990) - Stochastic Convenience Yield and the Pricing of Oil Contingent Claims. Journal of Finance; 45 (3), 959-976.

Hall, J., Nicholls, S. (2007) - Valuation of Mining Projects Using Option Pricing Techniques. JASSA; 4, 22-29.

Kamrad, B., Ernst, R. (2001) - An Economic Model for Evaluating Mining and Manufacturing Ventures with Output Yield Uncertainty. Operations Research; 49 (5), 690-699.

Kelly, S. (1998) - A Binomial Lattice Approach for Valuing a Mining Property IPO”. The Quarterly Review of Economics and Finance; 38 (3), 693-709.

Longstaff, F.A., Schwartz, E.S. (2001) - Valuing American Options by Simulation: A Simple Least Squares Approach". The Review of Financial Studies; 14 (1), 113-147.

Mahajan, A. (1990) - Pricing Expropriation Risk. Financial Management; 19 (4), 77-86.

Martzoukos, S.H. (2000) - Real Options with Random Controls and the Value of Learning. Annals of Operations Research; 99 (1), 305-323.

Moel, A., Tufano, P. (1999) - Bidding for the Antamina Mine: Valuation and Incentives in a Real Options Context in M. Brennan e L. Trigeorgis (eds.), Project Flexibility, Agency, and Competition, Oxford University Press, New York; 128-150.

Moyen, N., Slade, M., Uppal, R. (1996) -Valuing Risk and Flexibility: A Comparison of Methods. Resources Policy; 22 (1-2), 63-74.

Sagi, J.S. (1998) - The Interaction Between Quality Control and Production. SSRN Working Paper Series; disponível em http://ssrn.com/abstract=113769 (consultado em 30 de Setembro de 2010).

Schwartz, E.S. (1997) - The Stochastic Behavior of Commodity Prices: Implications for Valuation and Hedging. Journal of Finance; 52 (3), 923-973.

Schwartz, E.S., Trolle, A.B. (2010) - Pricing Expropriation Risk in Natural Resource Contracts: A Real Options Approach in W. Hogan e F. Sturzenegger (eds.), The Natural Resources Trap: Private Investment Without Public Commitment, MIT Press; 263-288.

Shafiee, S., Topal, E., Nehring, M. (2009) - Adjusted Real Option Valuation to Maximise Mining Project Value - A Case Study using Century Mine. Proceedings of the Project Evaluation Conference, The Australasian Institute of Mining and Metallurgy, Melbourne, 21-22 de Abril 2009.

Slade, M.E. (2001) - Valuing Managerial Flexibility: An Application of Real-Option Theory to Mining Investments. Journal of Environmental Economics and Management; 41 (2), 193-233. 
Tourinho, O. A. F. (1979) - The Valuation of Reserves of Natural Resources: An Option Pricing Approach. PhD Dissertation, University of California, Berkeley.

Trigeorgis, L. (1991) - A Log-Transformed Binomial Numerical Analysis Method for Valuing Complex Multi-Option Investments. Journal of Financial and Quantitative Analysis; 26 (3), 309-326.

Trigeorgis, L. (1996) - Real Options: Managerial Flexibility and Strategy in Resource Allocation. MIT Press, Cambridge, MA.

Trolle, A.B., Schwartz, E.S. (2009) - Unspanned Stochastic Volatility and the Pricing of Commodity Derivatives. The Review of Financial Studies; 22 (11): 4423-4461.

Tsekrekos, A.E, Shackleton, M.B., Wojakowski, R. (2003) - Evaluating Natural Resource Investments Using Least-Squares Monte Carlo Simulation Approach”. Proceedings of the 7th Annual International Conference on Real Options: Theory meets Practice, Washington D.C., 10-12 de Julho 2003. 